\title{
The Advantages of Spark Discharge Generation for Manufacturing of Nanoparticles with Tailored Properties
}

\author{
Maria E. Messing \\ Solid State Physics, NanoLund, Lund University, Box 118, 22100 Lund, Sweden \\ E-mail:maria.messing@ftf.lth.se
}

Received 5 November 2015; Accepted 3 June 2016;

Publication 1 August 2016

\begin{abstract}
The number of nanoparticle-based products on the market is expected to increase considerably during the coming decades. For this to happen a solid supply of high-quality nanoparticles is needed. For metal nanoparticles no large-scale manufacturing method exists today but production using spark discharge generation is believed to be one of the most suitable candidates. Spark discharge generation offers several advantages over other methods and the first steps towards scaling up have already been taken. In this paper the spark discharge generator concept is presented and its advantages are discussed. Examples of nanoparticle materials and applications for the as-generated particles are reviewed.
\end{abstract}

Keywords: Nanoparticles, spark discharge generation, aerosols.

\section{Introduction}

Smart nanomaterials are currently entering our everyday lives in various products and applications and this trend is expected to continue. At date nanomaterials can be found in a wide range of consumer products in categories such as food and beverage, electronics, clothing, sporting goods, cosmetics and personal care to mention some of them [1]. Nanoparticles in a wide range

Journal of Green Engineering, Vol. 5, 83-96.

doi: 10.13052/jge 1904-4720.5346

(C) 2016 River Publishers. All rights reserved. 
of sizes, shapes and compositions are building blocks for nanomaterials with an extensive range of applications. To establish a profitable and sustainable supply of nanoparticles industry is forced to use highly meticulous manufacturing of large amounts of material using cheap and environmentally friendly methods. In the manufacture of nanomaterials, it is also of utmost importance to ensure that no unintentional exposure to the produced material occurs due to the potential health risks of such exposure [2].

Several techniques can be used to manufacture nanoparticles from different materials. These are usually divided into chemical processing, mechanical milling or physical processing. In chemical processing, chemical precursors and solvents in liquid or gas form are used to form nanoparticles. These methods often require expensive chemicals, and can result in partly contaminated particles, and significant amounts of chemical waste [3]. Mechanical milling involves grinding a large piece of material into small particles. A very high energy input is required to produce small nanoparticles with this method, and it is difficult to manufacture particles with uniform size and shape [4].

Nanoparticle production by physical processes comprises sputtering and evaporation in the gas phase. Advantages of nanoparticle generation by physical processes includes avoidance of chemical waste, good control over particle size, generation of highly pure particles and a continuous generation process [5]. The physical process of flame spray pyrolysis (FSP) is a widely used method for manufacturing of metal oxide nanoparticles on an industrial scale [6]. FSP is however not the best choice for production of pure metal particles due to challenges with oxidation. Pure metal nanoparticles are instead often formed by material evaporation in an inert gas followed by subsequent nucleation and condensation [7]. This can be achieved in several ways, including furnace generation [8], glowing wire generation [9] or laser ablation [10]. These methods are, however, often not very energy efficient and massproduction might be problematic. A more energy efficient method to evaporate material is a spark discharge generator (SDG) where a plasma channel between two conducting electrodes (spark) heats and evaporates material [11]. The process is also referred to as spark ablation.

The SDG has a very simple design and consist of an electrode pair and a power supply. By placing several electrode pairs in parallel the generation process could be easily scaled up at low cost and with minimal impact on the environment, due to reasonable energy efficiency in the conversion of electrodes into particles and the possibility to recycle the non-heated carrier gas. In the EU-project BUONAPART-E, 21 partners from both industry and academia worked on this up-scaling to reach a production rate of $100 \mathrm{~kg}$ $\mathrm{d}^{-1}[12]$. 
In this paper the SDG will be briefly described (detailed descriptions can be found elsewhere [7, 9, 13-16]) and some of its advantages are discussed. It will also be explained how a further tailoring of nanoparticle properties can be achieved by connecting the SDG to other instruments commonly used in aerosol science. Finally, the nanoparticle materials produced by spark discharge generation and certain applications of these particles will be reviewed.

\section{Spark Discharge Generation}

\subsection{The Spark Discharge Generator}

The spark discharge generator consists of a chamber housing two opposing electrodes that are separated by a small gap (Figure 1). Nanoparticles are formed when material evaporated from the electrodes via the spark, is

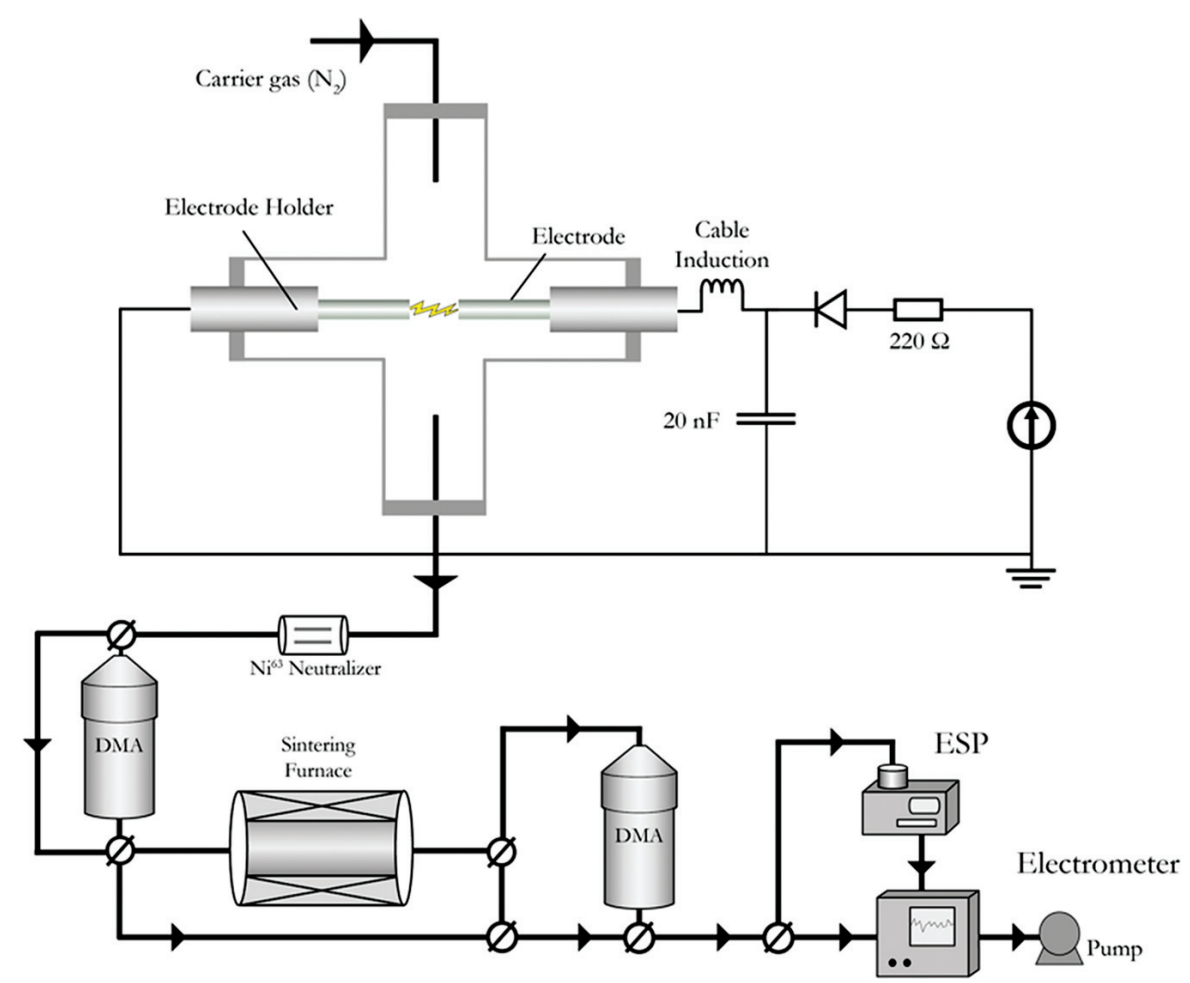

Figure 1 The basic components of the SDG, and the following aerosol instruments providing generation and deposition of nanoparticles with a controlled size and concentration onto any type of substrate. 
transported away from the heated gap region by means of a carrier gas. To create repeated spark discharges between the electrodes, a self-pulsed circuit is used, consisting of a capacitor bank driven by a high voltage DC power supply connected in parallel to the electrode gap. This could be modeled as a basic resistance, capacitance and inductance (RCL) circuit. The spark is formed ones the capacitor reaches the breakdown voltage of the carrier gas and discharges across the electrode gap. The energy of the spark discharge is approximately proportional to the capacitance and to the square of the discharge voltage, while the spark repetition frequency is proportional to the charging current. Each spark involves the formation of a plasma channel between the electrodes that is dominated by atoms and ions from the carrier gas. The single spark is an oscillatory event with a damping and duration determined by the total resistance, capacitance, and inductance in the circuit $[13,14]$ and influenced by the electrical properties of the electrode material.

Due to the high temperature of the plasma, the electrode surfaces are heated at local "hot spots" and a vapor plume is formed [16]. In addition, ions both from the anode, cathode and the carrier gas bombard the electrodes leading to further removal of electrode material [17]. When the vapor plume is cooled by adiabatic expansion and mixing with the carrier gas, nucleation will take place and atomic clusters are formed. These clusters will further grow into singlet particles that condense into primary particles that finally form agglomerate particles [16], as shown in Figure 2. The formation of these agglomerates can be directly affected and controlled by regulating several different parameters including the capacitance, the discharge frequency (by the output current), the electrode distance, the electrode material, the carrier gas flow rate, the type of carrier gas and the geometry of the chamber.

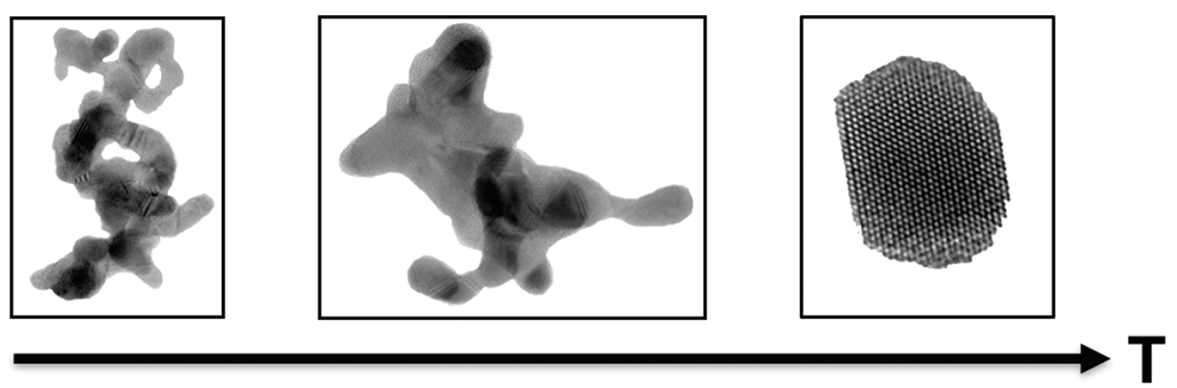

Figure 2 Transformation of the gold particle structure from an agglomerate to a compact particle by increasing temperature in the compaction tube furnace. 


\subsection{Tailoring of Nanoparticle Properties}

The particles resulting from the spark discharge generator are often chainlike agglomerate particles or sometimes compact particles with a very small diameter $(<10 \mathrm{~nm})$. For numerous applications compact particles with a larger size and of a narrow size distribution are needed. This can easily be achieved by connecting the spark discharge generator to commercially available instruments commonly used in aerosol science. An example of a possible setup is illustrated in Figure 1.

The agglomerate particles that are transported through the instruments via a carrier gas are first passed through a neutralizer in order to achieve a reproducible and known charge distribution of primarily uncharged and singly-charged particles [18]. Once the stable charge distribution is achieved, the charged particles are size-selected in a differential mobility analyzer (DMA). The DMA is a standard instrument in aerosol science, used to classify charged particles according to their mobility in an electric field [19]. If the particles are singly charged, their mobility can be related to the particle mobility diameter, $d_{m}$, which for spherical particles is equal to the geometrical particle diameter.

An agglomerated particle can be transformed into a compact, sphericallike particle by heating inside a compaction tube furnace [20] (Figure 2). The mobility diameter may thus decrease substantially due to the reduced drag force (increased mobility) of spherical particles compared to that of agglomerates, although the mass of the particles remains the same. If the particles are not reshaped, size distribution measurements can be performed directly on the agglomerated particles using an electrometer, provided that each particle passing through the DMA carries one single charge. A second DMA can be used to obtain size distributions of reshaped particles. Size distributions are obtained by scanning the voltage and measuring the resulting particle concentration with an electrometer.

The final aerosol instrument needed for controlled continuous deposition of particles onto a substrate is an electrostatic precipitator (ESP). The ESP focuses the charged particles onto a collector electrode [21], on which the substrate is placed, allowing high-efficiency deposition of particles with diameters up to about $200 \mathrm{~nm}$. Any type of substrate can be placed in the ESP even allowing depositions into liquids [22].

Connecting the SDG to the aerosol instruments as mentioned above enables a highly flexible system where nanoparticles with tunable size, shape, composition and concentration can be generated and deposited or collected. 
Other similar setups with slightly different aerosol instruments or other type of furnaces are also possible and enable generation of nanoparticles with different possibilities to tailor their properties.

\subsection{Advantages of the Method}

Compared to other methods to generate nanoparticles the SDG connected to commonly used aerosol instruments as described above are beneficial in a number of ways. One of the key advantages is the high quality products that are generated. Due to the inert carrier gas, the avoidance of chemicals and the on-line monitoring during manufacturing the nanoparticles have a high purity and a well defined size. Aggregation of the particles can also be easily avoided by adjusting the carrier gas flow. The same platform can be used for numerous different materials ranging from pure metals via metal oxides and alloys to semiconductor particles. Also metals that typically oxidize easily can be manufactured using this method. Since the heating is extremely local, the switching of materials can be fast, as opposed to furnace generation. The process is continuous, which enables delivery on demand and the possibility to post-process the particles while suspended in the carrier gas, which is requested for certain applications [23].

The next key advantage of this method is that it is environmentally friendly. Since the process requires no chemical precursors or additives, no chemical waste is produced nor needs to be handled. The only material being consumed is the bulk material (the electrodes) that is transformed into nanoparticles. The little unprocessed raw material can be recycled completely. The same is true for the inert carrier gas that is not consumed and that could be fully recycled. In addition, a life cycle analysis comparing nanoparticles produced by electrical discharge and chemical synthesis methods shows that the previous have a lower environmental impact, in most categories [24].

A third key advantage of this method is the reasonably cost-efficient manufacturing of nanoparticles. No costly precursors are needed and the process enables a comparably high energy efficiency in the conversion of electrodes into nanoparticles, especially if the size range below $10 \mathrm{~nm}$ is considered. The system can be scaled up by placing several SDG:s in parallel [25] which might permit a higher throughput at a lower cost per mass unit of produced nanoparticles. In addition, since the process is continuous, nanoparticles could be delivered on demand and hence costs related to over production can be avoided. 
The final key advantage of this method, utterly important during these times when the awareness of the potential health risks of nano-sized materials exposure is high, is the completely enclosed production system that prevents unintentional nanoparticle emissions. All generation, handing and deposition of nanoparticles take place in a closed system resulting in a very low probability of unwanted particle emissions.

\section{Materials and Application Examples}

\subsection{Materials}

Spark discharge generation is a highly versatile method for production of nanoparticles of a wide range of different materials. Soot-like carbon particles generated from graphite electrodes was reported already in the first description of the complete SDG concept [11]. Several groups have continued to generate carbon particles with the SDG [26-28] and even managed to produce radiolabelled soot-like carbon particles by soaking the electrode tip in a solution of the labelling material [29].

One of the most important classes of materials produced by the SDG method are metals, since no established method for mass production of metallic nanoparticles exists today but this method carries the potential for up-scaling. Metals that are not so prone to oxidation, including gold, silver, palladium, platinum, rhodium, and iridium have been produced by several groups [11, 13, 28, 30-35]. These metals have been generated under different gas atmospheres and in different types of SDG chambers/setups with some allowing amounts of oxygen and others having extensive gas purification and outgassing allowing no oxygen or water. In the latter type of systems, pure nanoparticles of metals that are more prone to oxidation, such as copper, iron, cobalt, tungsten, nickel, tin and antimony [13, 31, 35, 36] have been manufactured. Even a material such as magnesium that oxidises extremely easily has been produce as a pure metal with an SDG [37].

Another materials class that has been produced by the SDG method is metal oxides. This could often be achieved by allowing small amounts of oxygen during generation. If the carrier gas is not pure enough or the system is not completely leak-tight, metal oxide particles will form instead of pure metal particles for materials that are prone to oxidation. Examples of metal oxide nanoparticles generated by the SDG method are iron oxide, copper oxide, zinc oxide, cadmium oxide and titanium dioxide [28, 38-40]. 
Nanoparticles consisting of mixed elements, combinations of elements with different phases within the particle and alloys, have also been generated by the SDG method. This could be achieved either by using pre-alloyed/presintered electrodes or two electrodes of different materials. Examples include mixtures of carbon with iron, copper and zinc [39], copper and nickel [35], palladium mixed with platinum, gold and silver [33, 41, 42], chromium and cobalt [41] and magnesium with titanium [43]. Truly beneficial and uniquely reported in connection with this method is the possibility to manufacture nanoparticles of immiscible elements. Examples include antimony mixed with tin and copper [36], copper with silver and tungsten as well as platinum with gold [44].

Finally, as shown above, the SDG method can be used to generate nanoparticles of, in principle, any type of conducting material. The versatility when it comes to material capabilities does, however, not end there. Recently it was shown that also semiconductor particles could be generated using this method, silicon particles being the example [45].

\subsection{Examples of Applications}

Nanoparticles generated by the SDG method have been used for numerous applications in a wide variety of fields and listing them all would be an extensive task, outside the scope of this paper. A few examples of applications from three different fields will, however, be given. Both examples where the nanoparticles are deposited and where they are used directly in the gas phase will be presented. In addition, the specific properties, especially advantageous for each distinct application, will be mentioned.

In the field of environmental and nano safety research, where it is important to understand how nanoparticle emissions affect the climate and human health, SDG generated nanoparticles have been used in a number of studies. The great advantages of the SDG method in these fields are that it provides highly characterized nanoparticles with an extremely controlled concentration and an exposure situation mimicking the "real" situation where particles might be emitted to the air and inhaled [46]. For environmental studies, such as atmospheric pollution, it is mainly the soot-like particles that are of interest $[26,27]$. For inhalation studies a number of different nanoparticles generated by the SDG are potentially interesting and several studies have been performed $[29,38,40]$.

Nanoparticles generated by the SDG have also been used for studies of catalytic reactions and for hydrogen storage. For such applications the 
main advantages of the SDG method are the ability to produce exceedingly small particles, where the surface dominates, and to create particles of mixed elements. With the SDG method it is even possible to create atomic clusters beginning from a single atom [47]. Magnesium-based nanoparticles as well as palladium nanoparticles have been shown to be promising for hydrogen storage $[37,48]$. The synthesis of methane from carbon monoxide and hydrogen over nickel nanoparticles produced by the SDG method has been demonstrated and studied [49]. Also CO oxidation over SDG generated nanoparticles of palladium, rhodium, and palladium-silver have been studied $[32,34,42]$. From these studies it has been shown that using nanoparticles instead of the traditionally used single crystals can give slightly different results that might be closer to the "real situation" where catalysts consist of small nanoparticles.

A final example of a distinct application, where SDG generated nanoparticles have been used is for manufacturing of semiconductor structures $[3,30]$. Semiconductor nanowires, which are one-dimensional rod-like structures with high potential as building blocks for electronic, optoelectronic and sensor applications have been manufactured from gold nanoparticles deposited onto a substrate. In this case the nanoparticles act as seeds to initiate the (epitaxial) growth of the nanowires and determine their diameter and position on the substrate. The advantages of using the SDG method to generate the nanoparticles for this application includes manufacturing of particles that are monodisperse, of a high purity and completely spherical, giving reproducible conditions that is important for semiconductor materials [50]. The conventional method of semiconductor nanowire production is by epitaxial growth from nanoparticles deposited onto a substrate. To enable a more flexible process suitable for mass production of nanowires the aerotaxy method, where nanowires are grown directly in the gas phase from nanoparticle seeds, have been developed [8]. For this aerotaxy process the SDG method are considered to be one of the most suitable particle sources.

\section{Conclusions}

Nanoparticle production by the spark discharge generation method is one of the most promising methods for large scale manufacturing of metal nanoparticles. Compared to other methods it is environmentally friendly, reasonably cost-efficient and delivers high-quality nanoparticles with tailored properties. In addition, the process takes place in a completely enclosed production system, which prevents unintentional nanoparticle emissions that 
are potentially harmful to human health. Nanoparticles of a wide range of materials including metal, metal oxides, alloys and semiconductors can be manufactured. These nanoparticles have been used in a variety of different applications ranging from nanotoxicology studies, via hydrogen storage to manufacturing of semiconductor structures for electronic and optoelectronic applications. By placing several SDG units in parallel, the mass output can be greatly enhanced to meet the increasing demand of a robust supply of nanoparticles from producers of nanoparticle-based products.

\section{References}

[1] Vance, M. E., Kuiken, T., Vejerano, E. P., McGinnis, S. P., Hochella, M. F. Jr, Rejeski, D., et al. (2015). Nanotechnology in the real world: redeveloping the nanomaterial consumer products inventory. Beilstein J. Nanotechnol. 6, 1769-1780.

[2] Oberdorster, G., Oberdorster, E., and Oberdorster, J. (2005). Nanotoxicology: an emerging discipline evolving from studies of ultrafine particles. Environ. Health Perspect. 113, 823-839.

[3] Messing, M. E., Hillerich, K., Johansson, J., Deppert, K., and Dick, K. A. (2009). The use of gold for fabrication of nanowire structures. Gold Bull. 42, 172-181.

[4] Yadav, T. P., Yadav, R. M., and Singh, D. P. (2012). Mechanical milling: a top down approach for the synthesis of nanomaterials and nanocomposites. Nanosci. Nanotechnol. 2, 22-48.

[5] Biskos, G., Vons, V., Yurteri, C. U., and Schmidt-Ott, A. (2008). Generation and sizing of particles for aerosol-based nanotechnology. KONA Powder and Particle J. 26, 13-35.

[6] Wegner, K., and Pratsinis, S. E. (2003). Scale-up of nanoparticle synthesis in diffusion flame reactors. Chem. Eng. Sci. 58, 4581-4589.

[7] Meuller, B. O., Messinga, M. E., Engberga, D. L. J., Janssona, A. M., Johanssona, L. I. M., Norléna, S. M., et al. (2012). Review of spark discharge generators for production of nanoparticle aerosols. Aerosol Sci. Technol. 46, 1256-1270.

[8] Magnusson, M. H., Deppert, K., Malm, J. O., Bovin, J. O., and Samuelson, L. (1999). Size selected gold nanoparticles by aerosol technology. Nanostruc. Mater. 12, 45-48.

[9] Schmidt-Ott, A., Schurtenberger, P., and Siegmann, H. C. (1980). Enormous yield of photoelectrons from small particles. Phys. Rev. Lett. 45, 1284-1287. 
[10] Kato, M. (1976). Preparation of ultrafine particles of refractory oxides by gas evaporation method. J. App. Phys. 15, 757-760.

[11] Schwyn, S., Garwin, E., and Schmidt-Ott, A. (1988). Aerosol generation by spark discharge. J. Aerosol Sci. 19, 639-642.

[12] www.buonapart-e.eu

[13] Tabrizi, N. S., Ullmann, M., Vons, V. A., Lafont, U., and Schmidt-Ott, A. (2009). Generation of nanoparticles by spark discharge. J. Nanopart. Res. 11, 315-332.

[14] Hontnón, E., Palomares, J. M., Stein, M., Guo, X., Engeln, R., Nirschl, H., et al. (2013). The transition from spark to arc discharge and its implications with respect to nanoparticle production. J. Nanopart. Res. 15:1957.

[15] Itina, T. E., and Voloshko, A. (2013). Nanoparticle formation by laser ablation in air and by spark discharges at atmospheric pressure. Appl. Phys. B, 113, 473-478.

[16] Pfeiffer, T. V., Feng, J., and Schmidt-Ott, A. (2014). New developments in spark production of nanoparticles. Adv. Powder. Technol. 25, 56-70.

[17] Wagner, M., Kohut, A., Geretovszky, Zs., Seipenbusch, M., and Galbács, G. (2016). Observation of fine-ordered patterns on electrode surfaces subject to extensive erosion in a spark discharge. J. Aerosol Sci. 93, 16-20.

[18] Wiedensohler, A. (1988). An approximation of the bipolar chargedistribution for particles in the sub-micron range. J. Aerosol Sci. 19, 387-389.

[19] Knutson, E. O., and Whitby, K. T. (1975). Aerosol classification by electron mobility: Apparatus, theory, and applications. J. Aerosol Sci. $6,443-451$.

[20] Schmidt-Ott, A. (1988). In situ measurement of the fractal dimensionality of ultrafine aerosol particles. Appl. Phys. Lett. 52:954.

[21] Deppert, K., Schmidt, F., Krinke, T., Dixkens, J., and Fissan, H. (1996). Electrostatic precipitator for homogeneous deposition of ultrafine particles to create quantum-dot structures. J. Aerosol Sci. 27, S151-S152.

[22] Svensson, C. R., Messing, M. E., Lundqvist, M., Schollin, A., Deppert, K., Pagels, J. H., et al. (2013). Direct deposition of gas phase generated aerosol gold nanoparticles into biological fluids - corona formation and particle size shifts. PLOS One 8:74702.

[23] Heurlin, M., Magnusson, M. H., Lindgren, D., Ek, M., Wallenberg, L. R., Deppert, K., et al. (2012). Continuous gas-phase synthesis of nanowires with tunable properties. Nature 492, 90-94. 
[24] Slotte, M., Metha, G., and Zevenhoven, R. (2015). Life cycle indicator comparison of copper, silver, zinc and aluminum nanoparticle production through electric arc evaporation or chemical reduction. Int. J. Energy Environ. Eng. 6, 233-243.

[25] Stein, M., and Kruis, F. E. (2015). Scale-up of metal nanoparticle production. Paper Presented at TechConnect World Innovation Conference \& Expo, Washington, DC.

[26] Helsper, C., Molter, W., Löffler, F., Wadenpohl, C., Kaufmann, S., and Wenninger, G. (1993). Investigations of a new aerosol generator for the production of carbon aggregate particles. Atmos. Environ. A 27, 1271-1275.

[27] Horvath, H., and Gangl, M. (2003). A low-voltage spark generator for production of carbon particles. J. Aerosol Sci. 34, 1581-1588.

[28] Roth, C., Ferron, G. A., Karg, E., Lentner, B., Schumann, G., Takenaka, S., et al. (2004). Generation of ultrafine particles by spark discharging. Aerosol Sci. Tech. 38, 228-235.

[29] Brown, J. S., Kim, C. S., Reist, P. C., Zeman, K. L., and Bennett, W. D. (2000). Generation of radiolabeled "soot-like" ultrafine aerosols suitable for use in human inhalation studies. Aerosol Sci. Tech. 32, 325-337.

[30] Messing, M. E., Dick, K. A., Wallenberg, L. R., and Deppert, K. (2009). Generation of size-selected gold nanoparticles by spark discharge - for growth of epitaxial nanowires. Gold Bull. 42, 20-26.

[31] Seipenbusch, M., Weber, A. P., Schiel, A., and Kasper, G. (2003). Influence of the gas atmosphere on restructuring and sintering kinetics of nickel and platinum aerosol nanoparticle agglomerates. J. Aerosol Sci. 34, 1699-1709.

[32] Messing, M. E., Westerström, R., Meuller, B. O., Blomberg, S., Gustafson, J., Andersen, J. N., et al. (2010). Generation of Pd model catalyst nanoparticles by spark discharge. J. Phys. Chem. C 114, 9257-9263.

[33] Byeon, J. H., Park, J. H., and Hwang, J. H. (2008). Spark generation of monometallic and bimetallic aerosol nanoparticles. J. Aerosol Sci. 39, 888-896.

[34] Blomberg, S., Westerström, R., Martin, N. M., Lundgren, E., Andersen, J. N., Messing, M. E., et al. (2014). A high pressure X-ray photoelectron spectroscopy study of oxidation and reduction of $\mathrm{Rh}(100)$ and $\mathrm{Rh}$ nanoparticles. Surf. Sci. 628, 153-158.

[35] Guo, X., Wagner, M., Gutsche, A., Meyer, J., Seipenbusch, M., and Nirschl, H. (2015). Laboratory SWAXS combined with a low-pressure 
impactor for quasi-online analysis of nanoparticles generated by spark discharge. J. Aerosol. Sci. 85, 17-29.

[36] Lafont, U., Simonin, L., Tabrizi, N. S., Schmidt-Ott, A., and Kelder, E. M. (2009). Synthesis of nanoparticles of $\mathrm{Cu}, \mathrm{Sb}, \mathrm{Sn}, \mathrm{SnSb}$ and $\mathrm{Cu}_{2} \mathrm{Sb}$ by densification and atomization process. J. Nanosci. Nanotechnol. 9, 2546-2552.

[37] Vons, V.A., Anastasopol, A., Legerstee, W. J., Mulder, F. M., Eijt, S. W. H., and Schmidt-Ott, A. (2011). Low-temperature hydrogen desorption and the structural properties of spark discharge generated $\mathrm{Mg}$ nanoparticles. Acta Mater. 59, 3070-3080.

[38] Evans, D. E., Harrison, R. M., and Ayres, J. G. (2003). The Generation and characterization of metallic and mixed element aerosols for human challenge studies. Aerosol Sci. Tech. 379, 75-987.

[39] Kim, J. T., and Chang, J. S. (2005). Generation of metal oxide aerosol particles by a pulsed spark discharge technique. J. Electroststics 63, 911-916.

[40] Kreyling, W. G., Biswas, P., Messing, M. E., Gibson, N., Geiser, M., Wenk, A., et al. (2011). Generation and characterization of stable, highly concentrated titanium dioxide nanoparticle aerosols for rodent inhalation studies. J. Nanopart. Res. 13, 511-524.

[41] Tabrizi, N. S., Xu, Q., van der Pers, N. M., Lafont, U., and Schmidt-Ott, A. (2009). Synthesis of mixed metallic nanoparticles by spark discharge. J. Nanopart. Res. 11, 1209-1218.

[42] Blomberg, S., Gustafson, J., Martin, N. M., Messing, M. E., Deppert, K., Liu, Z., et al. (2013). Generation and oxidation of aerosol deposited PdAg nanoparticles. Surf. Sci. 616, 186-191.

[43] Anastasopol, A., Pfeiffer, T. V., Middelkoop, J., Lafont, U., CanalesPerez, R. J., Schmidt-Ott, A., et al. (2013). Reduced enthalpy of metal hydrid formation for $\mathrm{Mg}$-Ti nanocomposites produced by spark discharge generation, JACS 135, 7891-7900.

[44] Tabrizi, N. S., Xu, Q., van der Pers, N. M., and Schmidt-Ott, A. (2010). Generation of mixed metallic nanoparticles from immiscible metals by spark discharge. J. Nanopart. Res. 12, 47-259.

[45] Vons, V. A., de Smet, L. C. P. M., Munao, D., Evirgen, A., Kelder, E. M., and Schmidt-Ott, A. (2011). Silicon nanoparticles produced by spark discharge. J. Nanopart. Res. 13, 4867-4879.

[46] Messing, M. E., Svensson, C. R., Pagels, J., Meuller, B. O., Deppert, K., and Rissler, J. (2013). Gas-borne particles with tunable and highly 
controlled characteristics for nanotoxicology studies. Nanotoxicology 7 , 1052-1063.

[47] Maisser, A., Barmpounis, K., Attoui, M. B., Biskos, G., and Schmidt-Ott, A. (2015). Atomic cluster generation with an atmospheric spark discharge generator, Aerosol Sci. Technol. 49, 886-894.

[48] Vons, V. A., Leegwater, H., Legerstee, W. J., Eijt, S. W. H., and SchmidtOtt, A. (2010). Hydrogen storage properties of spark generated palladium nanoparticles. Int. J. Hyd. Energy 35, 5479-5489.

[49] Weber, A. P., Seipenbusch, M., Thanner, C., and Kasper, G. (1999). Aerosol Catalysis on Nickel Nanoparticles. J. Nanopart. Res. 1, 253-265.

[50] Messing, M. E., Hillerich, K., Bolinsson, J., Storm, K., Johansson, J., Dick, K. A., et al. (2010). A comparative study of the effect of gold seed particle preparation method on nanowire growth. Nano Res. 3, 506-519.

\section{Biography}

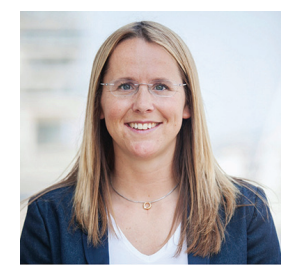

M. E. Messing is currently working as an assistant professor at NanoLund, one of the strategic research areas of Lund University, Lund, Sweden. She obtained her Ph.D. degree from the division of Solid State Physics, also at Lund University, in 2011. Her area of interest includes generation, characterization and applications of nanoparticles generated by physical methods. 